\title{
Impact of Partial Sleep Deprivation on Psychological Functioning: Effects on Mindfulness and Basic Psychological Need Satisfaction
}

\author{
Rachel Campbell $^{1}$ (D) Bart Soenens $^{1} \cdot$ Netta Weinstein $^{2} \cdot$ Maarten Vansteenkiste $^{1}$
}

(C) Springer Science+Business Media, LLC 2017

\begin{abstract}
Extending previous research on the psychological costs of sleep deprivation, the present study examined the impact of insufficient sleep on the capacity to be mindful as well as on the satisfaction of individuals' basic psychological needs, two psychological sources of mental health. The interrelationship between these two psychological resources and fatigue following sleep deprivation was also examined. Participants were 49 adults (77\% female; Mage $=32.81$ years, $\mathrm{SD}=13.09$ years) who were randomly assigned to either an experimental $(N=23)$ or a control $(N=26)$ group. The study had a 4-day within-person design. In the experimental group, a baseline assessment day was followed by 3 days of partial sleep deprivation (i.e., 5-h sleep per night), whereas participants in the control group slept as usual across the 4-day period. Participants rated their fatigue and psychological functioning each evening and wore an actigraph watch which monitored their sleep. Participants reported increased fatigue after 1 day of sleep deprivation, whereas it took 3 days of sleep deprivation before their mindfulness and need satisfaction deteriorated. Mediational analyses indicated that decreased need satisfaction after 3 days of sleep deprivation was completely accounted for (i.e., explained) by increased fatigue and subsequent decreases in mindfulness. These findings build on previous research by showing that mindfulness and need-based experiences not only precede but also follow from sleep at night.
\end{abstract}

Rachel Campbell

Rachel.Campbell@UGent.be

1 Department of Developmental, Personality and Social Psychology, Ghent University, Henri Dunantlaan 2, 9000 Ghent, Belgium

2 School of Psychology, Cardiff University, Cardiff, Wales
Keywords Sleep deprivation $\cdot$ Mindfulness $\cdot$ Basic psychological needs $\cdot$ Fatigue

Sleep deprivation is rampant in modern society. Although it is recommended that adults sleep 7 hours or more per night to function optimally (Watson et al. 2015), recent polls and studies indicate that up to $40 \%$ of the general public sleep less than 6 hours a night on average (Ford et al. 2015; Jones 2013). Insufficient sleep can have a detrimental impact on physical functioning, with fatigue being one of the most immediate manifestations of sleep deprivation (e.g., Klumpers et al. 2015; Minkel et al. 2014). Apart from fatigue, lack of sleep also comes at a considerable psychological cost and has been shown to result in depressed mood (Kahn-Greene et al. 2007), elevated anxiety (Pires et al. 2016), and impaired cognitive functioning (e.g., Frenda and Fenn 2016). The present study sought to further investigate the psychological effects of sleep deprivation on two important and interrelated predictors of well-being, that is, individuals' capacity to be mindful and the satisfaction of their basic psychological needs for autonomy, competence, and relatedness (Brown and Ryan 2003; Ryan and Deci 2017).

Mindfulness is conceptualized as an open awareness of present moment experiences (Brown and Ryan 2003). There is rising interest in mindfulness in the health literature because an increasing number of studies indicate that being mindful has salutary effects on psychological (e.g., Chiesa and Serretti 2009; Vollestad et al. 2012) and physical health (e.g., Davidson et al. 2003; Reibel et al. 2001). More relevant to the present research, a number of correlational (e.g., Howell et al. 2010, 2008) and mindfulness-based intervention studies (e.g., Kanen et al. 2015) indicate that being mindful leads to better sleep quality and longer sleep duration. This is presumably because mindfulness allows for a more observant and accepting approach to sleep-interfering arousal processes 
which promotes better sleep at night (Lundh 2005). However, although a lack of mindfulness might leave individuals vulnerable to poor sleep, the reverse is also plausible, namely that being sleep deprived may interfere with individuals' capacity to be mindful. Yet, no previous studies have directly examined this alternative causal pathway.

Previous experimental research provides some indirect evidence which suggests that insufficient sleep may undermine the capacity to be mindful. For instance, experimental studies have found that sleep deprivation leads to a lack of focused attention (Harrision and Horne 2000), with partial sleep deprivation (i.e., sleeping 5 hours a night) increasing individuals' distractibility during monotonous tasks (Anderson and Horne 2006). Furthermore, a more recent experimental study found that total sleep deprivation (i.e., total sleep loss for 24 hours) resulted in increased mind wandering (i.e., having task-unrelated thoughts) during a subsequent visual task (Poh et al. 2016). Given that mindfulness involves purposefully paying attention to events and experiences as they occur (Brown and Ryan 2003), these findings imply that mindfulness is likely to be impaired by sleep deprivation.

Importantly, the open awareness characteristic of mindful individuals is thought to facilitate behavior that is concordant with one's preferences, values, and interests (Ryan and Deci 2017; Christie et al. 2016) and mindfulness has previously been linked to the fulfillment of inherent psychological needs (Brown and Ryan 2003). Indeed, given its close ties with mindfulness, another important aspect of psychological functioning that may be impacted by sleep deprivation is the satisfaction of basic psychological needs. Self-determination theory (SDT; Ryan and Deci 2017) identifies three basic psychological needs which are thought to be universal and essential for mental health. These are the need for autonomyexperiencing a sense of volition and choice in one's activities, the need for competence-feeling capable of achieving desired outcomes, and the need for relatedness - feeling connected to, and cared for, by important others. Whereas need satisfaction is important for wellbeing and optimal functioning, the active frustration (i.e., experiencing pressure, incompetence, loneliness) of these needs is said to elicit maladaptive functioning (Vansteenkiste and Ryan 2013).

These three basic psychological needs are central to individuals' well-being, psychosocial adjustment, and physical health (Ryan and Deci 2017). Studies have demonstrated their satisfaction to relate to psychological wellbeing, including life satisfaction, positive emotions, and subjective energy (Chen et al. 2015; Deci and Ryan 2000; Reis et al. 2000), whereas their frustration has been shown to predict ill-being and to deplete energetic resources (e.g., Bartholomew et al. 2011). Several studies have also found a relation between SDT's psychological needs and quality and quantity of sleep among healthy adults (Campbell et al. 2015) and clinical samples at risk for poor sleep (Campbell et al. 2016; Campbell et al. 2017a). Interestingly, findings from a recent diary study among adolescents indicated that poorer self-reported quality and quantity of sleep related to more need frustration throughout the day (Campbell et al. 2017b). However, the correlational nature of these findings prevented conclusions about the direction of effects.

Indeed, given that sleep deprivation reduces subjective energy (e.g., Frenda and Fenn 2016), and that energy is presumably required to remain present and attentive to daily experiences (i.e., mindful), insufficient sleep is likely to undermine psychological need satisfaction. Thus, reduced mindfulness resulting from depleted energy is likely to preclude individuals from selecting need-satisfying activities as well as prevent them from deriving a sense of need satisfaction from ongoing activities throughout the day. There is some indirect evidence for our reasoning, with studies indicating that mindfulness relates positively to need satisfaction and that, conversely, low mindfulness increases individuals' susceptibility for need frustrating experiences (e.g., Brown and Ryan 2003). However, research is yet to formally test the possibility that sleep deprivation causally impacts on daily need experiences through increases in fatigue, which then in turn impairs present moment awareness (i.e., reduced mindfulness). Furthermore, given that energy is needed to proactively engage in and seek opportunities for need satisfaction, it is also possible that reduced energy following sleep deprivation (i.e., fatigue) may directly undermine psychological need satisfaction. Thus, fatigue may directly result in lower need satisfaction/more need frustration (i.e., without being accounted for by impaired mindfulness).

The main objective of the present study was to examine the impact of partial sleep deprivation (i.e., sleeping less than 5 hours a night) on subjective fatigue, mindfulness, and psychological need-based experiences. We hypothesized that sleep deprivation would result in increased fatigue, impaired mindfulness, and reduced need-based experiences (as indexed by reduced need satisfaction and increased need frustration; i.e., hypothesis 1). Apart from examining the main effect of sleep deprivation, our second objective was to explore in greater detail how these hypothesized changes in fatigue and psychological functioning (i.e., mindfulness and need experiences) relate to one another. We examined whether increased fatigue stemming from sleep deprivation would lead to poorer psychological functioning. Specifically, we hypothesized that increases in fatigue following sleep deprivation would relate to decreased need satisfaction and increased need frustration both directly and indirectly via reduced mindfulness (i.e., hypothesis 2). 


\section{Method}

\section{Participants}

All participants were recruited via flyers and online advertisements through social media. The final sample consisted of 49 Belgian adults (see Fig. 1 for a flow chart displaying the selection process for these participants, and a full description of this process below). The mean age of the sample was 32.81 years $(\mathrm{SD}=13.09$, range $=21-$ 69 years). Sixty-seven percent of the final sample was female; $53 \%$ were employed and the remaining $47 \%$ were unemployed or students.

\section{Procedure}

The study consisted of two parts. In the first part, participants were screened using an online questionnaire and in the second part, the experimental study was conducted. Prior to filling in the online questionnaire (i.e., part 1), all participants were made aware of the voluntary nature of the study and their anonymity was guaranteed. Participants were informed that they may be asked to deprive their sleep in the second part of the study. Online informed consent was obtained from all participants. Participants who only completed the first part of the study received one cinema ticket for their participation and participants who completed both parts received two cinema tickets. The study was carried out between February 2016 and May 2016 and was approved by the host University's Ethical Review Board.

\section{Part 1 (Screening)}

An online questionnaire was used to screen participants for the following inclusion criteria: participants were required to be older than 18 years of age, could not have children under the age of three, use sleep medication, and have severe sleep disturbances or depressive symptoms, given the likely impact of these factors on sleep. Of the 114 individuals who filled in the online screening, $58(50.88 \%)$ met the inclusion criteria and were invited to participate in part 2 . Of the 58 participants who were invited to participate, $4(6.90 \%)$ declined because they were not willing to deprive their sleep. The remaining 54 participants were randomly allocated to the experimental group $(N=26)$ and the control group $(N=28$; Fig. 1$)$.

\section{Part 2 (Experimental Study)}

The study involved a 4-day within-person design. In the experimental group, participants were instructed to sleep as usual on the first day of the study and on the following three consecutive days, they were instructed to restrict their sleep to 5 hours per night, whereas participants in the control group were simply instructed to sleep as usual for the 4 days. Participants in the experimental group were free to choose when they wanted to sleep at night (e.g., between 00:00 and 5:00 or between 02:00 and 07:00). All participants took part from a Sunday evening to a Thursday morning to avoid weekend effects (Ryan, Bernstein, \& Brown 2010). Participants were allowed to choose which month they wanted to participate in the study (either February, March, April, or May) and were informed whether they would be required to deprive

Fig. 1 Flow chart of participation

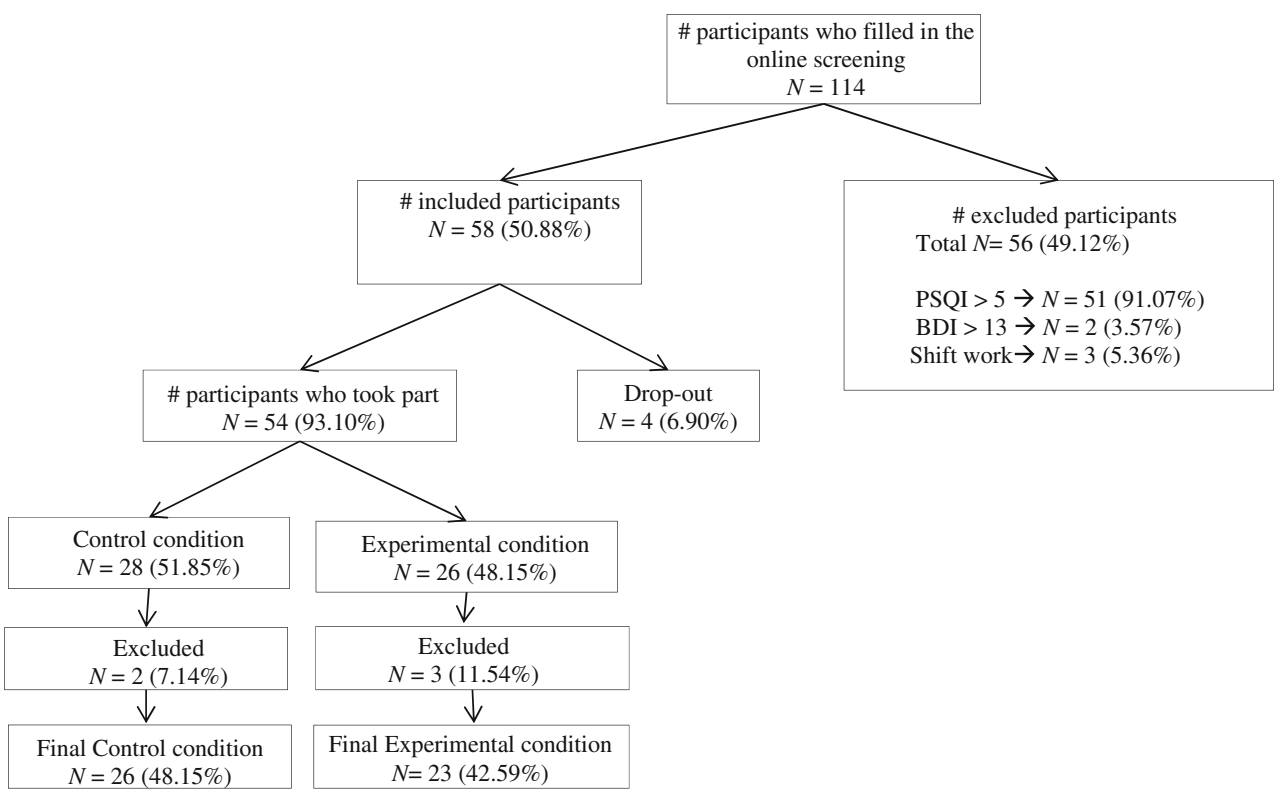


their sleep or not on the first day of the study (i.e., on the Sunday morning). During a first home visit, trained research assistants provided participants with a diary and an actigraph watch. Participants in both groups filled in the diary every evening and rated items assessing their fatigue and psychological functioning (i.e., mindfulness and psychological need experiences). All participants also wore the actigraph watch for the full duration of the study which objectively monitored their sleep duration. During a second home visit, the completed diaries and actigraph watches were collected by the research assistants.

After preliminary inspection of the data, results from five participants were deemed invalid and were removed from the data set. These were data from three participants from the experimental group who failed to comply with the sleep restriction protocol and two participants from the control group who slept less than 5 hours per night throughout the duration of the study. This resulted in a final sample of 23 in the experimental group and 26 in the control group (total $n=49$ ).

\section{Measures}

Pittsburgh Sleep Quality Index The Pittsburgh Sleep Quality Index (PSQI) (Buysse et al. 1989) was used to screen participants for sleep disturbances. The PSQI consists of 19 items which generate scores on 7 components: subjective poor sleep quality, sleep latency, sleep duration, habitual sleep efficiency, sleep disturbances, use of hypnotics, and daytime dysfunction. These component scores are then summed to produce a global score between 0 and 21. A global PSQI score of $>5$ distinguishes between poor and good sleepers (Buysse et al. 1989), with higher scores indicating worse sleep quality. All participants who initially scored above 5 were excluded from participating in the experimental phase of the study to ensure a healthy and homogenous sample. Of the 114 participants who completed the screening measure, $44.74 \%(n=51)$ were excluded due to scoring above the cut-off of 5 .

Beck Depression Inventory-II The Beck Depression Inventory-II (BDI-II) (Beck et al. 1996) screened participants for depressive symptoms. Participants were asked to rate the 21 depressive symptoms on a scale from 0 (not present) to 3 (severe) with respect to how they felt during the past week. Scores were summed to provide an overall score between 0 and 63. BDI scores from 0 to 13 suggest absent to minimal depressive symptoms, whereas scores from 14 to 63 represent mild to severe depressive symptoms. Two participants (1.74\% of the sample) who scored above 13 were excluded from participating in the study.

Fatigue Fatigue was assessed using the lassitude subscale from the Inventory of Depression and Anxiety Symptoms (IDAS; Watson et al. 2007). This six-item scale was adapted to assess symptoms of fatigue experienced during the past day (e.g., "Today I felt sleepy and drowsy"). All items were rated on a 5-point Likert scale ranging from 1 (not at all) to 5 (very much so). The scale had an average reliability of .87 ; range across days $=.84-.89$.

Mindfulness State mindfulness was measured using a validated shortened version of the Mindfulness Attention Awareness Scale (MAAS; Brown and Ryan 2003). This version consists of five items (i.e., "Today I said or did things on 'automatic pilot' without being conscious of what I did or said") which assessed the extent to which participants were mindful during the past day on a scale of 1 (completely disagree) to 6 (completely agree). The MAAS had an average reliability of .74 , range across days $=.68-.77$.

Psychological Need Experiences Daily satisfaction and frustration of the basic psychological needs for autonomy, competence, and relatedness were assessed using the Basic Psychological Need Satisfaction and Need Frustration Scale (BPNSNFS; Chen et al. 2015). The scale consists of 24 items, 8 items per need, 4 of which tap into need satisfaction and 4 of which tap into need frustration. All participants rated on a scale of 1 (not at all true) to 5 (very true) as to whether they felt that their needs for autonomy (e.g., "Today I felt that my decisions reflected what I really wanted", "I felt forced to do many things that I didn't choose to do"), competence (e.g., "Today I felt capable of achieving my goals", "I had serious doubts about whether I could do things well"), and relatedness (e.g., "Today I felt connected with the people who care about me and who I care about", "Today I felt excluded from the group that I want to belong to") were satisfied or frustrated during the past day. The diary format of the BPNSNFS has shown good validity in past research (Van der Kaap-Deeder et al. 2017). Two composite scores were created by averaging the 12 items assessing need satisfaction (average $\alpha=.91$; range across days $=.90-.91$ ) and the 12 items that assessed need frustration (average $\alpha=.87$; range across days $=.85-.89)$.

\section{Objective Registration of Sleep Duration}

To objectively register daily sleep duration, all participants wore a MotionWatch 8 actigraph watch (Wave Medical B.V.; The Netherlands) for the full duration of the experimental study. The MotionWatch 8 is an unobtrusive, light-weight device which was worn by all participants on their nondominant arm. The device includes a digital accelerometer which measures movement, thereby allowing for the differentiation between wake and sleep states for each 30 -s period of recording. Daily sleep duration was extracted from the actigraph data using the CamNtech MotionWare software (version1.1.25) validated algorithm. 


\section{Data Analysis}

To examine whether the induced sleep deprivation had an effect on the study variables (i.e., hypothesis 1), repeated measures ANOVAs were performed using SPSS. In these analyses, we examined whether shifts in the assessed constructs from one measurement moment to another differed in the control versus the experimental condition. Specifically, for each dependent variable (i.e., fatigue, mindfulness, need satisfaction, and need frustration), condition (i.e., control group versus experimental group) was included as a between-person factor and time was included as a within-person factor. Specifically, the following comparisons were made: (1) baseline assessment versus sleep deprivation day 1, (2) baseline assessment versus sleep deprivation day 2, and (3) baseline assessment versus sleep deprivation day 3 . We chose to examine effects of sleep deprivation in this stepwise fashion (rather than through one omnibus effect across the days) so as to gain insight into exactly when (i.e., on which day) the effects of the sleep deprivation manifested.

Having established effects of sleep deprivation on changes in each of the separate study variables, we then examined the integrated model (i.e., hypothesis 2). To test this model, we estimated within-person changes in each of the study variables through latent change models (LCMs) in Mplus 7 (with maximum likelihood as estimator). LCMs are widely acknowledged as a more reliable method to estimate change compared to difference scores. Specifically, they estimate within-person change across two measurement moments (e.g., from the baseline assessment to sleep deprivation day 3) using latent variables for intercept (i.e., level) and slope (i.e., change over time) (Beyers and Goossens 2008). Each latent change model consisted of a longitudinal measurement model defining the latent variables (i.e., fatigue, mindfulness, need satisfaction, and need frustration) at each time point by their respective indicators and a structural model which defined latent level and change factors for each latent variable and further specified how these levels and changes were interrelated (Hertzog et al. 2003). Further, co-variances among the residuals of the same indicators over time were specified (Sörbom 1975). In the longitudinal measurement model, each latent variable was represented by two parcels. Parcels were created by combining stronger loading items with weaker loading items from each scale (Little et al. 2002).

The latent factor scores for the level (i.e., intercept) and within-person changes in each variable were extracted and saved as separate variables. These saved variables were then used to test the proposed integrated models in Mplus7. Specifically, a structural model was used to examine whether within-person decreases in fatigue resulting from sleep deprivation would lead to decreases in mindfulness, and whether decreases in mindfulness would then in turn lead to impaired need experiences (i.e., hypothesis 2 ). Model fit was evaluated using the Comparative Fit Index (CFI); the root mean squared error of approximation (RMSEA); and the standardized root means square residual (SRMR). An acceptable fit was indicated by CFI values of .90 or above, and RMSEA and SRMR values of around .08 or below (Hu and Bentler 1999; Kline 2005). Background characteristics (i.e., age and gender) were controlled for in all models.

\section{Results}

The relations between participants' background characteristics (i.e., age and gender) and the study variables were examined using a MANCOVA with gender as a between-subjects variable, age as a covariate, and all the study variables as dependent variables. Neither participants' age, $F(19$, $24)=1.69$, ns, or gender, $F(19,24)=.12$, ns, yielded a significant multivariate main effect.

To examine whether participants in the experimental group slept fewer hours relative to their baseline assessment and relative to the control group, a series of repeated measures ANOVAs were performed with objectively assessed sleep duration as an outcome. As shown in Table 1 , all time $\times$ condition interactions were significant, indicating that the experimental group and control group displayed different trajectories in sleep duration. Furthermore, as shown in Table 2, the mean scores indicated that participants in the experimental group slept less than those in the control group and averaged $4: 38,4: 38$, and $4: 30$ hours of sleep on sleep deprivation days 1,2 , and 3 , respectively. These findings indicate that the experimental manipulation of sleep duration was successful.

Main Effects of Sleep Deprivation In Table 1, the condition effects, time effects, and time $\times$ condition interactions are shown for each of the assessed study variables. Significant time $\times$ condition interactions indicate that the control group and experimental group displayed a different trajectory in the assessed outcomes from the baseline assessment to the sleep deprivation days. As this is most relevant to our research question, we will limit ourselves to discussing the findings of these interactions.

With regard to fatigue, all time $\times$ condition interactions were significant. This indicated that relative to the control group, participants in the sleep deprivation condition reported significantly more fatigue already after 1 day of sleep deprivation, with this effect becoming stronger across the following 2 additional days of sleep deprivation. In contrast, with regard to psychological functioning (i.e., mindfulness and needbased experiences), the time $\times$ condition interactions were only significant after 3 days of sleep deprivation. This indicated that it took 3 days of sleep deprivation before participants in the experimental group reported significantly reduced mindfulness and lower need satisfaction, relative to both the 
Table 1 Repeated measures mixed model ANOVA

\begin{tabular}{|c|c|c|c|c|c|c|c|c|c|c|c|c|c|c|c|c|c|c|}
\hline \multirow{3}{*}{$\begin{array}{l}\text { Baseline } \\
\text { versus sleep } \\
\text { deprivation }\end{array}$} & \multicolumn{6}{|c|}{ Condition } & \multicolumn{6}{|l|}{ Time } & \multicolumn{6}{|c|}{ Condition $\times$ time } \\
\hline & \multicolumn{2}{|c|}{$\mathrm{BL}-\mathrm{SD} 1$} & \multicolumn{2}{|c|}{$\mathrm{BL}-\mathrm{SD} 2$} & \multicolumn{2}{|c|}{$\mathrm{BL}-\mathrm{SD} 3$} & \multicolumn{2}{|c|}{$\mathrm{BL}-\mathrm{SD} 1$} & \multicolumn{2}{|c|}{$\mathrm{BL}-\mathrm{SD} 2$} & \multicolumn{2}{|l|}{$\mathrm{BL}-\mathrm{SD} 3$} & \multicolumn{2}{|c|}{$\mathrm{BL}-\mathrm{SD} 1$} & \multicolumn{2}{|c|}{$\mathrm{BL}-\mathrm{SD} 2$} & \multicolumn{2}{|c|}{$\mathrm{BL}-\mathrm{SD} 3$} \\
\hline & $F$ & $\eta^{2}$ & $F$ & $\eta^{2}$ & $F$ & $\eta^{2}$ & $F$ & $\eta^{2}$ & $F$ & $\eta^{2}$ & $F$ & $\eta^{2}$ & $F$ & $\eta^{2}$ & $F$ & $\eta^{2}$ & $F$ & $\eta^{2}$ \\
\hline \multicolumn{19}{|c|}{ Manipulation check } \\
\hline $\begin{array}{l}\text { Objective } \\
\text { sleep } \\
\text { duration } \\
\text { Daily measures }\end{array}$ & $35.31 * *$ & .43 & $29.76^{* *}$ & .39 & $41.30 * *$ & .47 & $70.50 * *$ & .60 & $88.26^{* *}$ & .65 & $101.82 * *$ & .68 & $55.93 * *$ & .54 & $40.99 * *$ & .47 & $75.75 * *$ & .62 \\
\hline Fatigue & .04 & .00 & 1.99 & .05 & $5.26 *$ & .10 & $4.53 *$ & .10 & $20.79 * *$ & .31 & $21.38 * *$ & .32 & $9.60 * *$ & .18 & $40.19 * *$ & .46 & $41.88 * *$ & .47 \\
\hline Mindfulness & .15 & .00 & .30 & .01 & $3.95^{*}$ & .08 & 2.48 & .05 & 1.01 & .02 & .45 & .01 & .28 & .01 & .61 & .01 & $8.18 * *$ & .15 \\
\hline $\begin{array}{l}\text { Need } \\
\text { satisfaction }\end{array}$ & 1.94 & .04 & 2.94 & .06 & $5.11 *$ & .10 & $4.96^{*}$ & .10 & $4.69^{*}$ & .09 & 1.02 & .02 & .18 & .00 & .19 & .00 & $4.39 *$ & .09 \\
\hline $\begin{array}{l}\text { Need } \\
\text { frustration }\end{array}$ & 2.28 & .05 & 2.23 & .05 & $5.01 *$ & .10 & 1.22 & .03 & .09 & .00 & 1.57 & .03 & .32 & .01 & .21 & .00 & .55 & .01 \\
\hline
\end{tabular}

$B L$ baseline assessment, $S D 1$ sleep deprivation day 1, SD2 sleep deprivation day 2, SD3 sleep deprivation day 3

$* p<.05 ; * *<.01$

Table 2 Means and standard deviations for all study variables from the control group and the experimental group

\begin{tabular}{|c|c|c|}
\hline Variable (unit) & $\begin{array}{l}\text { Control group } \\
\text { Mean (SD) }\end{array}$ & $\begin{array}{l}\text { Experimental group } \\
\text { Mean (SD) }\end{array}$ \\
\hline \multicolumn{3}{|c|}{ Objective sleep quantity (min) } \\
\hline Baseline & $423.81(52.07)$ & $416.70(58.29)$ \\
\hline Sleep deprivation day 1 & $415.81(65.54)$ & $278.30(18.04)$ \\
\hline Sleep deprivation day 2 & $397.69(56.29)$ & $278.83(29.05)$ \\
\hline Sleep deprivation day 3 & $413.00(54.62)$ & $270.26(19.63)$ \\
\hline \multicolumn{3}{|l|}{ Fatigue $(1-5)$} \\
\hline Baseline & $1.99(.85)$ & $1.73(.77)$ \\
\hline Sleep deprivation day 1 & $1.89(.95)$ & $2.25(.77)$ \\
\hline Sleep deprivation day 2 & $1.77(.74)$ & $2.61(.78)$ \\
\hline Sleep deprivation day 3 & $1.72(.66)$ & $2.91(.94)$ \\
\hline \multicolumn{3}{|l|}{ Mindfulness (1-5) } \\
\hline Baseline & $4.06(.75)$ & $4.04(.70)$ \\
\hline Sleep deprivation day 1 & $3.96(.73)$ & $3.84(.64)$ \\
\hline Sleep deprivation day 2 & $4.04(.74)$ & $3.86(.69)$ \\
\hline Sleep deprivation day 3 & $4.30(.63)$ & $3.66(.69)$ \\
\hline \multicolumn{3}{|l|}{ Need satisfaction (1-5) } \\
\hline Baseline & $4.16(.58)$ & $3.92(.56)$ \\
\hline Sleep deprivation day 1 & $4.04(.57)$ & $3.84(.59)$ \\
\hline Sleep deprivation day 2 & $4.06(.59)$ & $3.77(.56)$ \\
\hline Sleep deprivation day 3 & $4.21(.52)$ & $3.76(.59)$ \\
\hline \multicolumn{3}{|l|}{ Need frustration (1-5) } \\
\hline Baseline & $1.53(.51)$ & $1.76(.59)$ \\
\hline Sleep deprivation day 1 & $1.50(.54)$ & $1.68(.41)$ \\
\hline Sleep deprivation day 2 & $1.54(.58)$ & $1.72(.46)$ \\
\hline Sleep deprivation day 3 & $1.38(.41)$ & $1.73(.56)$ \\
\hline
\end{tabular}

control group and their baseline assessment. Of note, none of the time $\times$ condition interactions were significant for need frustration, indicating that the experimental manipulation did not cause an increase in participants' experienced need frustration. These findings are reflected in the mean scores for each variable across both conditions, which are shown in Table 2.

Explanatory Chain of Mechanisms A SEM model was tested to examine the hypothesized chain of mechanisms following sleep deprivation, that is, whether changes in fatigue would relate to changes in mindfulness which, in turn, would account for (i.e., explain) the relationship between sleep deprivation and reduced need satisfaction. Given that effects were only found on all variables after 3 days of sleep deprivation, we focused on changes from the baseline assessment to sleep deprivation day 3 in these models. Also, because sleep deprivation did not affect need frustration, need frustration was no longer included in these models. Paths were modeled from the experimental contrast (i.e., control group versus experimental group) to change in fatigue, from change in fatigue to change in mindfulness, and from change in mindfulness to change in need satisfaction. Furthermore, the baseline levels of all variables were controlled for. Results of this model, shown in Fig. $2, X^{2} / \mathrm{df}=.71, \mathrm{CFI}=.99$, RMSEA $=.04$, SRMR $=.08$, indicated that 3 days of sleep deprivation contributed to an increase in fatigue, which in turn related to a decrease in mindfulness, which then in turn related to reduced need satisfaction. Next, direct paths were added from the experimental contrast to change in mindfulness and need satisfaction and from change in fatigue to change in need satisfaction, but these paths were dropped because they were non-significant 


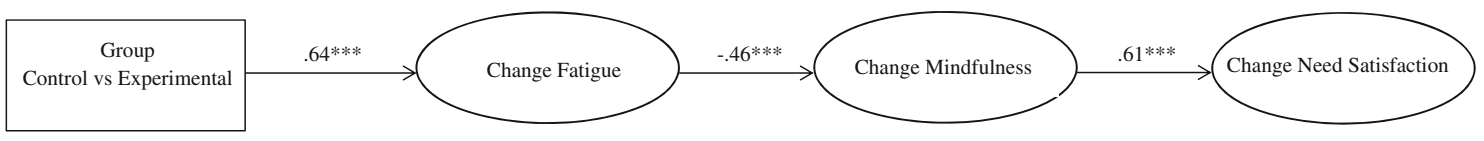

Note. $* p<.05, * * p<.01, p * * *<.001$.

All changes shown are from the baseline assessment to sleep deprivation day 3 .

Fig. 2 Experimental contrast predicting changes in need satisfaction via changes in fatigue and mindfulness

and adding them did not improve model fit. Finally, the indirect association between the experimental sleep deprivation and reduced need satisfaction via increased fatigue and reduced mindfulness was significant $(\beta=-.18, p<.01 ; \mathrm{CI}$ $95 \%[-.285 ;-.075])$.

\section{Supplementary Analysis}

In supplementary analyses, we considered two alternative sequences. First, we explored whether reduced psychological functioning due to sleep deprivation would also contribute to increased fatigue, in line with the nascent literature suggesting causal effects of psychological need satisfaction on subjective energy (e.g., Martela and Ryan 2016). That is, we considered the possibility that reductions in mindfulness following sleep deprivation would undermine need-based experiences which then, in turn, would predict further increases in fatigue.

Specifically, this model defined paths from the experimental manipulation to change in mindfulness, from change in mindfulness to change in need satisfaction, and lastly, from change in need satisfaction to change in fatigue (all of these represented changes from baseline to day 3 ). Results revealed that sleep deprivation contributed to reduced mindfulness $(\beta=-.38, p<.001)$, which in turn related to reduced need satisfaction $(\beta=.58, p<.001)$; however, the relation between change in need satisfaction and change in fatigue was nonsignificant $(\beta=.05, \mathrm{~ns})$. Rather, results indicated that reduced mindfulness yielded a direct relation with increased fatigue $(\beta=-.43, p<.001)$, which was not accounted for by reductions in need satisfaction. This non-significant path between change in need satisfaction and fatigue was dropped from the final model, $X^{2} / \mathrm{df}=1.05, \mathrm{CFI}=.99, \mathrm{RMSEA}=.03$, $\mathrm{SRMR}=.07$. Notably, the effect of mindfulness on increased fatigue emerged above and beyond the effect of the experimental sleep deprivation on increased fatigue $(\beta=.49$, $p<.001$ ), indicating that reduced mindfulness only partially accounted for the association between sleep deprivation and fatigue. The indirect associations between the experimental sleep deprivation and reduced need satisfaction $(\beta=-.22$, $p<.01$; CI 95\% $[-.338 ;-.083])$ and increased fatigue $(\beta=.15, p<.001 ;$ CI 95\% $[.080 ; 237])$ via reduced mindfulness were both significant. Overall, the initially hypothesized model (Fig. 2) appeared to represent the data more parsimoniously than this alternative sequence of events.

Second, although in our initial model (Fig. 2) we assumed that impaired mindfulness would precede decreases in need satisfaction, the reverse sequence is also plausible. Namely, because decreased need satisfaction may result in less present moment awareness, impaired mindfulness may also result from decreased need satisfaction. Indeed, findings from a recent longitudinal study among employees found need satisfaction to predict higher levels of state mindfulness across months (Olafsen 2017). Thus, we also examined whether increased fatigue following sleep deprivation would predict decreased need satisfaction which, in turn, would undermine mindfulness.

In this second supplementary model, we added paths from the experimental manipulation to change in fatigue, from change in fatigue to change in need satisfaction, and lastly, from change in need satisfaction to change in mindfulness (similar to the previous models, all changes represented shifts from baseline to day 3 ). Results revealed that sleep deprivation contributed to increased fatigue $(\beta=.64, p<.001)$; however, similar to the previous model tested, the relation between change in fatigue and change in need satisfaction was nonsignificant $(\beta=-.28, \mathrm{~ns})$. Decreased need satisfaction did, however, relate to impaired mindfulness $(\beta=.37, p<.001)$. Results further revealed that the experimental contrast continued to yield a direct relation with impaired mindfulness $(\beta=-.31, p<.001)$. The fit of this model, $X^{2} / \mathrm{df}=2.28$, $\mathrm{CFI}=.91, \mathrm{RMSEA}=.16, \mathrm{SRMR}=.08$, was poorer than the fit of our original model with impaired mindfulness predicting decreased need satisfaction (i.e., Fig. 2). Thus, again, it appeared that our initially hypothesized model better represented the data. Overall, these findings indicated that increased fatigue following sleep deprivation contributed to decreased need satisfaction to the extent that it related to impairments in individuals' capacity to be mindful.

\section{Discussion}

A sizeable percentage of the general public sleep less than 6 hours a night on average (Ford et al. 2015; Jones 2013), putting their functioning at considerable risk. Extending previous 
research which found sleep deprivation to have a range of psychological consequences, including mood disturbance (e.g., Kahn-Greene et al. 2007) and cognitive dysfunction (Frenda and Fenn 2016), the present research examined the effects of insufficient sleep on two important and interrelated resources of mental health, namely the capacity to be mindful and the satisfaction of individuals' basic psychological needs. Furthermore, we considered a chain of mechanisms to understand the effect of sleep deprivation, thereby examining whether increased fatigue would relate to reduced mindfulness, which then, in turn, would forestall need satisfaction.

Replicating a number of previous studies, the present research found sleep deprivation to have an immediate impact on subjective energy (e.g., Klumpers et al. 2015), with the effects of insufficient sleep on increased fatigue already manifesting after one night of sleep deprivation. In contrast, it took longer for effects on psychological functioning to appear, with participants only reporting reduced mindfulness and decreased need satisfaction after 3 days of being sleep deprived. Interestingly, the induced sleep deprivation had no effect on participants' experienced need frustration, indicating that 3 days of being partially sleep deprived did not serve to actively frustrate individuals' basic psychological needs. Perhaps, if the manipulation of sleep duration had been prolonged across more days or had been more intense during the 3-day period (e.g., less than 4 hours of sleep or total sleep loss for 24 hours), the observed effects on psychological functioning would have been more pronounced and may even have resulted in increased need frustration. For example, if sleep had been deprived across more days, individuals may have begun to feel pressured and unable to handle daily tasks and challenges and perhaps also experience more interpersonal conflict and difficulties, signifying need frustration. In line with this, previous research found that sleeping less than 5 hours across 7 consecutive days had cumulative and escalating effects on waking functioning (Dinges et al. 1997).

The present results extend previous work which indicated that both mindfulness (e.g., Howell et al. 2010; Kanen et al. 2015) and need-based experiences (e.g., Campbell et al. 2015; Campbell et al. 2017a, 2017b) are predictive of quality and quantity of sleep, by showing that sleep at night, and sleep duration in particular, can also yield a recursive impact on these psychological factors. Similarly, findings from a recent diary study also found daily quality and quantity of sleep to contribute to daily need-based experiences (Campbell et al. 2017b). However, these previous findings were correlational in nature and did not allow for conclusions about the direction of effects. Overall, this body of research indicates that a cyclical effect may exist, wherein sleep deprivation undermines psychological functioning, which in turn leads to further sleep reductions.

The present findings are important because they speak to the dynamic interface between individuals' physiological and psychological needs, an issue that has received very little prior attention. Also, the damaging impact of sleep deprivation on need satisfaction is worrisome from a well-being perspective. Indeed, a large body of work indicates that the costs of low need satisfaction to physical and psychological well-being are numerous and long-lasting; for example, reductions in psychological need satisfaction have been linked to lower life satisfaction, fewer positive emotions, more depression and anxiety (see review in Ryan and Deci 2017), and physiological arousal including greater salivary cortisol and blood pressure (Quested et al. 2011; Weinstein et al. 2016). The results of the present findings suggest that accumulated sleep deprivation might indirectly lead to these adverse outcomes because it undermines psychological need satisfaction.

We also examined whether the observed reductions in subjective energy and mindfulness helped to explain why participants in the experimental group reported decreased need satisfaction after 3 days of sleep deprivation. In line with our hypothesized model, results revealed that reduced energy following sleep deprivation related to impaired mindfulness which, in turn, contributed to decreased need satisfaction. Although we explored the possibility that reduced energy would relate to decreased need satisfaction directly (i.e., without being accounted for by impaired mindfulness), this direct path was non-significant. The finding that reduced mindfulness led to lower need satisfaction is in line with previous research which found that individuals low in mindfulness report less need satisfaction (Brown and Ryan 2003; Campbell et al. 2015); whereas in previous research, mindfulness was examined as an individual difference characteristic. Here, we found that variations were present from day to day.

There are several possible reasons why reduced impaired present moment awareness on a given day predicts reduced need satisfaction. When low in mindfulness, individuals may be less able to effectively and wholeheartedly engage in daily activities and thus be less able to derive need satisfaction from these activities. In addition, impaired mindfulness likely leaves individuals less in tune with their interests and values (Christie et al. 2016), and as a result, they may less proactively select and engage in potentially need-satisfying activities, and may also be less responsive to opportunities for need satisfaction throughout the day. Furthermore, given that mindfulness has been shown to mitigate emotional reactivity (Ortner et al. 2007), it is also possible that reduced mindfulness may undermine people's capacity to effectively regulate negative emotions that stem from encountered need-frustrating experiences, which may aggravate the resulting need frustration.

In a supplementary analysis, we explored an alternative sequence, namely whether participants' reported impaired psychological functioning after 3 days of sleep deprivation would contribute to further increases in fatigue. Estimation of this alternative model showed that sleep deprivation affected fatigue directly as well as indirectly, through reduced 
mindfulness. This suggests that, although fatigue follows to some extent from a lack of sleep itself, there are also psychological mechanisms at play that may exacerbate the experienced fatigue. That is, when low in mindfulness, people may become more easily distracted by their thoughts and emotions during ongoing activities and it may then require energy for them to re-center themselves into the present moment, which in itself may be draining. Overall, these results suggest that impaired subjective energy following sleep deprivation (i.e., fatigue) may not only be predictive of but may also be predicted by impaired mindfulness.

\section{Limitations and Directions for Future Research}

The present study had some limitations. First, as a result of our effort to create a homogeneous, well-functioning sample, the final sample was fairly small and consisted mainly of women. The sample was also subject to self-selection bias, as all individuals knew before agreeing to participate that there was a possibility that they would be required to deprive their sleep; those who may have coped even worse with sleep deprivation might have self-selected out of the study. If this is the case, we might expect the detrimental impact of sleep deprivation to be even stronger in the general population. Furthermore, because all participants were screened for sleep disturbances and depressive symptoms, the sample was relatively healthy and may not generalize well to clinical populations. Thus, it would be interesting to examine whether these results can be replicated among larger, more heterogeneous samples and perhaps also among individuals who are used to routinely sleeping less, such as shift workers. In addition, subjective energy and psychological functioning were only assessed once each evening. Future research could use experience sampling methodology (e.g., Shiffman et al. 2008) to collect multiple daily assessments using a smartphone application (e.g., Runyan et al. 2013) in order to provide an understanding of how these processes are related within the day. For example, it might be that costs to psychological functioning are seen more in the afternoons and evenings, when initial energy levels have been depleted. Future experimental studies could also examine the effect of insufficient sleep on more varied indicators of cognitive functioning such as decision making, creative thinking, or problem solving, and examine whether these effects are explained by impaired mindfulness following sleep deprivation. In addition, future experimental studies could examine whether manipulating sleep quality has a similar or more pronounced effect on psychological functioning, by for example, waking participants up at regular intervals throughout the night (e.g., Finan et al. 2015) to disrupt sleep continuity. Finally, in light of the present findings, future studies could examine whether participation in a low-dose mindfulness intervention (e.g.,
Hülsheger et al. 2015) protects participants against the detrimental effects of sleep deprivation.

Author Contributions RC: Assisted with the design, coordinated the data collection, performed the data analysis, and wrote the paper. BS: Assisted with data analysis and critically revised the paper. NW: Assisted with data analysis and critically revised the paper. MV: Designed the study, assisted with data analysis, and critically revised the paper.FundingThis study was funded by Fund for Scientific Research Flanders (grant number FWO.OPR.2013. 0140.01 - IV2).

\section{Compliance with Ethical Standards}

Conflict of Interest The authors declare that they have no conflict of interest.

Ethical Approval All procedures performed in studies involving human participants were in accordance with the ethical standards of Ghent University and with the 1964 Helsinki declaration and its later amendments or comparable ethical standards. This article does not contain any studies with animals performed by any of the authors.

Informed Consent Informed consent was obtained from all individual participants included in the study.

\section{References}

Anderson, C., \& Horne, J. A. (2006). Sleepiness enhances distraction during a monotonous task. Sleep, 29, 573-576.

Bartholomew, K. J., Ntoumanis, N., Ryan, R. M., \& ThøgersenNtoumani, C. (2011). Psychological need thwarting in the sport context: assessing the darker side of athletic experience. Journal of Sport \& Exercise Psychology, 33, 75-102.

Beck, A. T., Steer, R. A., \& Brown, G. K. (1996). Manual for the Beck Depression Inventory-II. San Antonio: Psychological Corporation.

Beyers, W., \& Goossens, L. (2008). Dynamics of perceived parenting and identity formation in late adolescence. Journal of Adolescence, 31, 165-184. https://doi.org/10.1016/j.adolescence.2007.04.003.

Brown, K. W., \& Ryan, R. M. (2003). The benefits of being present: mindfulness and its role in psychological well-being. Journal of Personality and Social Psychology, 84, 822-848.

Buysse, D. J., Reynolds, C. F., Monk, T. H., Berman, S. R., \& Kupfer, D. J. (1989). The Pittsburgh Sleep Quality Index - a new instrument for psychiatric practice and research. Psychiatry Research, 28, 193213.

Campbell, R., Tobback, E., Delesie, L., Vogelaers, D., Mariman, A., \& Vansteenkiste, M. (2017a). Basic psychological need experiences, fatigue, and sleep in individuals with unexplained chronic fatigue. Stress \& Health.

Campbell, R., Vansteenkiste, M., Delesie, L. M., Mariman, A. N., Soenens, B., Tobback, E., Van der Kaap-Deeder, J., \& Vogelaers, D. P. (2015). Examining the role of psychological need satisfaction in sleep: a self-determination theory perspective. Personality and Individual Differences, 77, 199-204.

Campbell, R., Vansteenkiste, M., Delesie, L. M., Soenens, B., Tobback, E., Vogelaers, D., \& Mariman, A. (2016). The role of psychological need satisfaction, sleep, and mindfulness in the health related quality of life of people living with HIV. Journal of Health Psychology.

Campbell, R., Vansteenkiste, M., Soenens, B., Vandenkerckhove, B., \& Mouratidis, A. (2017b). Examining daily variation in adolescent sleep: the role of psychological need experiences. Manuscript submitted for publication. 
Chen, B., Vansteenkiste, M., Beyers, W., Boone, L., Deci, E. L., Duriez, B., Lens, W., Matos, L., Mouratidis, A., Ryan, R. M., Sheldon, K. M., Soenens, B., Van Petegem, S., Van der Kaap-Deeder, J., \& Verstuyf, J. (2015). Basic psychological need satisfaction, need frustration, and need strength across four cultures. Motivation and Emotion, 39, 216-236.

Chiesa, A., \& Serretti, A. (2009). Mindfulness-based stress reduction for stress management in healthy people: a review and meta-analysis. Journal of Alternative and Complimentary Medicine, 15, 593-600.

Christie, A. M., Atkins, P. W. B., \& Donald, J. (2016). The meaning and doing of mindfulness: the role of values in the link between mindfulness and well-being. Mindfulness, 8, 368-378.

Davidson, R. J., Kabat Zinn, J., Schumacher, J., Rosenkranz, M., Muller, D., Santorelli, S. F., \& Sheridan, J. F. (2003). Alterations in brain and immune function produced by mindfulness meditation. Psychosomatic Medicine, 65, 564-570.

Deci, E. L., \& Ryan, R. M. (2000). The 'what' and 'why' of goal pursuits: human needs and the self determination of behavior. Psychological Inquiry, 11, 227-268.

Dinges, D. F., Pack, F., Williams, K., Gillen, K. A., Powell, J. W., Ott, G. E., Aptowicz, C., \& Pack, A. I. (1997). Cumulative sleepiness, mood disturbance, and psychomotor vigilance performance decrements during a week of sleep restricted to $4-5$ hours per night. Sleep, 20, 267-277.

Finan, P. H., Quartana, P. J., \& Smith, M. T. (2015). The effects of sleep continuity disruption on positive mood and sleep architecture in healthy adults. Sleep, 38, 1735-1742.

Ford, E. S., Cunningham, T. J., \& Croft, J. B. (2015). Trends in selfreported sleep duration among US adults from 1985 to 2012. Sleep, 38, 829-832.

Frenda, S. J., \& Fenn, K. M. (2016). Sleep less, think worse: the effect of sleep deprivation on working memory. Journal of Applied Research in Memory and Cognition, 5, 463-469.

Harrision, Y., \& Horne, J. A. (2000). The impact of sleep deprivation on decision making: a review. Journal of Experimental Psychology: Applied, 6, 236-249.

Hertzog, C., Dixon, R. A., Hultsch, D. F., \& MacDonald, S. W. S. (2003). Latent change models of adult cognition: are changes in processing speed and working memory associated with changes in episodic memory? Psychology and Aging, 18, 755-769. https://oi.org/10. 1037/0882-7974.18.4.755.

Howell, A. J., Digdon, N. L., \& Buro, K. (2010). Mindfulness predicts sleep-related self-regulation and well-being. Personality and Individual Differences, 48, 419-424.

Howell, A. J., Digdon, N. L., Buro, K., \& Sheptycki, A. R. (2008). Relations among mindfulness, well-being, and sleep. Personality and Individual Differences, 45, 773-777.

Hu, L., \& Bentler, P. M. (1999). Cutoff criteria for fit indexes in covariance structure analysis: conventional criteria versus new alternatives. Structural Equation Modeling, 6, 1-55.

Hülsheger, U. R., Feinholdt, A., \& Nübold, A. (2015). A low-dose mindfulness intervention and recovery from work: effects on psychological detachment, sleep quality, and sleep duration. Journal of Occupational and Organizational Psychology, 88, 464-489.

Jones, J. (2013), December 5-8 In U.S., 40\% get less than recommended amount of sleep. Retrieved from http:// http://www.gallup.com/poll/ 166553/less-recommended-amount-sleep.aspx.

Kahn-Greene, E. T., Killgore, D.B., Kamimori, G.H., Balkin, T.J., \& Killgore, W.D.S. (2007). The effects of sleep deprivation on symptoms of psychopathology in healthy adults. Sleep Medicine, 8, 215221.

Kanen, W. J., Nazir, R., Sedky, K., Pradhan, K., \& Basant. (2015). The effects of mindfulness-based interventions on sleep disturbance: a meta-analysis. Adolescent Psychiatry, 5, 105-115.

Kline, R. B. (2005). Principles and practice of structural equation modeling. New York: Guilford Press.
Klumpers, U. M. H., Veltman, D. J., Van Tol, M. J., Kloet, R. W., Boellaard, R., Lammerstsma, A. A., \& Hoogendijk, W. J. G. (2015). Neurophysiological effects of sleep deprivation in healthy adults, a pilot study. PLoS One, 10, e0116906.

Little, T. D., Cunningham, W. A., Shahar, G., \& Widaman, K. F. (2002). To parcel or not to parcel: exploring the question, weighing the merits. Structural Equation Modeling, 9, 151-173.

Lundh, L.-G. (2005). The role of acceptance and mindfulness in the treatment of insomnia. Journal of Cognitive Psychotherapy, 19, 29-39.

Martela, F., \& Ryan, R. M. (2016). Prosocial behavior increases wellbeing and vitality even without contact with the beneficiary: causal and behavioral evidence. Motivation and Emotion, 40, 351-357.

Minkel, J., Moreta, M., Muto, J., Htaik, O., Jones, C., Basner, M., \& Dignes, D. (2014). Sleep deprivation potentiates HPA axis stress reactivity in healthy adults. Health Psychology, 33, 1430-1434.

Olafsen, A. (2017). The implications of need-satisfying work climates on state mindfulness in a longitudinal analysis of work outcomes. Motivation and Emotion, 41, 22-37.

Ortner, C. N. M., Kilner, S. J., \& Zelazo, P. D. (2007). Mindfulness meditation and reduced emotional interference on a cognitive task. Motivation and Emotion, 31, 271-283.

Pires, G. N., Bezerra, A. G., Tufik, S., \& Andersen, M. L. (2016). Effects of acute sleep deprivation on state anxiety levels: a systematic review and meta-analysis. Sleep Medicine, 24, 109-118.

Poh, J. H., Chong, P. L. H., \& Chee, M. W. L. (2016). Sleepless night, restless mind: effects of sleep deprivation on mind wandering. Journal of Experimental Psychology: General, 145, 1312-1318.

Quested, E., Bosch, J. A., Burns, V. E., Cumming, J., Ntoumanis, N., \& Duda, J. L. (2011). Basic psychological need satisfaction, stressrelated appraisals, and dancers' cortisol and anxiety responses. Journal of Sport \& Exercise Psychology, 33, 828-846.

Reibel, D., Greeson, J., Brainard, G., \& Rosenzweig, S. (2001). Mindfulness-based stress reduction and health-related quality of life in a heterogeneous patient population. General Hospital Psychiatry, 23, 183-192.

Reis, H. T., Sheldon, K. M., Gable, S. L., Roscoe, J., \& Ryan, R. M. (2000). Daily well-being: the role of autonomy, competence, and relatedness. Personality and Social Psychology Bulletin, 26, 419435.

Runyan, J., Steenbergh, T., Bainbridge, C., Daugherty, D., Oke, L., \& Fry, B. (2013). A smartphone ecological momentary assessment intervention "App" for collecting real-time data and promoting selfawareness. PLOS/one. https://doi.org/10.1371/journal.pone. 0071325.

Ryan, R. M., \& Deci, E. L. (2017). Self-determination theory: basic psychological needs in motivation, development, and wellness. New York: Guilford.

Ryan, R. M., Bernstein, J.H., \& Brown, K. W. (2010). Weekends, work and well-being: Psychological need satisfactions and day of the week effects on mood, vitality, and physical symptoms. Journal of Social and Clinical Psychology, 29, 95-122.

Shiffman, S., Stone, A., \& Hufford, M. (2008). Ecological momentary assessment. Annual Review of Clinical Psychology, 4, 1-32.

Sörbom, D. (1975). Detection of correlated errors in longitudinal data. British Journal of Mathematical and Statistical Psychology, 28, 138-151.

Van der Kaap-Deeder, J., Vansteenkiste, M., Soenens, B., \& Mabbe, E. (2017). Children's daily well-being: the role of mothers', teachers', and siblings' autonomy support and psychological control. Developmental Psychology, 53(2), 237.

Vansteenkiste, M., \& Ryan, R. M. (2013). On psychological growth and vulnerability: basic psychological need satisfaction and need frustration as a unifying principle. Journal of Psychotherapy Integration, 23, 263-280. 
Vollestad, J., Nielsen, M. B., \& Nielsen, G. H. (2012). Mindfulness and acceptance based interventions for anxiety disorders: a systematic review and meta-analysis. British Journal of Clinical Psychology, 51, 239-260.

Watson, N. F., Badr, M. S., Belenky, G., Bliwise, D. L., Buxton, O. M., Buysse, D., Dinges, D. F., Gangwisch, J., Grandner, M. A., Kushida, C., Malhotra, R. K., Martin, J. L., Patel, S. R., Quan, S. F., \& Tasali, E. (2015). Recommended amount of sleep for a healthy adult: a joint consensus statement of the American Academy of Sleep Medicine and Sleep Research Society. Sleep, 38, 843-844.
Watson, D., O'Hara, M. W., Simms, L. J., Kotov, R., \& Chmielewski, M. (2007). Development and validation of the Inventory of Depression and Anxiety Symptoms (IDAS). Psychological Assessment, 19, 253-268.

Weinstein, N., Legate, N., Kumashiro, M., \& Ryan, R. M. (2016). Autonomy support and diastolic blood pressure: long term effects and conflict navigation in romantic relationships. Motivation and Emotion, 40, 212-225. 